\title{
TWISTED FOURIER-MUKAI TRANSFORMS AND BUNDLES ON NON-KÄHLER ELLIPTIC SURFACES
}

\author{
VASILE BRÎnzăNEscu AND RuxANDRA MORARU
}

\begin{abstract}
In this paper, we study holomorphic rank-2 vector bundles on non-Kähler elliptic surfaces. Our main tool for analysing these bundles is of course the spectral cover. However, given the non-Kähler condition, the elliptic surfaces we are considering do not have sections and gerbes naturally arise in this context. The spectral construction presented in this paper is a modification of the Fourier-Mukai transform for elliptic fibrations without a section. After examining some of the properties of this FourierMukai transform, we give a complete classification of vector bundles on these surfaces.
\end{abstract}

\section{Introduction}

The study of sheaves over elliptic fibrations has been a very active area of research in both mathematics and physics over the past fifteen years; this is partly due to the role that such fibrations play in both mirror symmetry and the theory of integrable systems. An object that has, more recently, proven very useful in their study is the (relative) Fourier-Mukai transform; this transform is an equivalence of derived categories of sheaves on elliptic fibrations with a section whose properties are by now well-understood [Mu, BBRP, B, BM]. Consider, for example, a smooth elliptic fibration with a section $Y \rightarrow B$ that does not have singular fibres; if $\mathcal{E}$ is a locally free sheaf on $Y$, then under some mild assumptions, the transform assigns to $\mathcal{E}$ a torsion sheaf on the relative Jacobian $J(Y)$ of $Y$, which is supported on its spectral cover. An important point, which makes this construction possible, is the existence of a universal sheaf on $Y \times_{B} J(Y)$. However, if the elliptic fibration $Y \rightarrow B$ does not have a section, then there is no universal sheaf on $Y \times{ }_{B} J(Y)$; what exists instead is a twisted universal sheaf [C1], which can be used to define a "twisted" Fourier-Mukai transform that is now an equivalence of derived categories of twisted sheaves [C2, DP].

In this article, we consider holomorphic vector bundles on a specific class of fibrations without a section, non-Kähler elliptic surfaces. Although bundles on projective elliptic fibrations have been extensively studied, not very much is known about the non-Kähler case; another motivation for investigating bundles on these surfaces comes from recent developments in superstring theory, where six-dimensional non-Kähler manifolds occur in the context of type IIA string compactifications with non-vanishing background $H$-field - in fact, all the non-Kähler examples appearing in the physics literature so far are non-Kähler principal elliptic fibrations (see [BBDG, CCFLMZ, GP]

Received by the editors September 2, 2003. Revised January 28, 2005.

The first author was partially supported by Swiss NSF contract SCOPES 2000-2003, No.7 IP 62615 and by contract CERES 39/2002-2004. $32 \mathrm{~J} 15$.

2000 Mathematics Subject Classification. Primary: 14J60; Secondary: 14D22, 14F05, 14J27, 
and the references therein). We first construct a particular twisted Fourier-Mukai transform for locally free sheaves on non-Kähler principal elliptic bundles, transforming them into torsion sheaves, which has the advantage of allowing us to work with sheaves instead of twisted sheaves; then, we use it to obtain a classification of rank-2 vector bundles on arbitrary non-Kähler elliptic surfaces (the existence and stability properties of such bundles are examined in [BrMo1, BrMo2]). Note that this transform also makes sense for coherent sheaves, but since we are primarily interested in classifying vector bundles, we restrict our definition to locally free sheaves. Furthermore, the techniques developed here naturally extend to the case of sheaves on higher dimensional non-Kähler elliptic and torus fibrations.

A minimal non-Kähler elliptic surface $X$ is a Hopf-like surface that admits a holomorphic fibration $\pi: X \rightarrow B$, over a smooth connected compact curve $B$, whose smooth fibres are isomorphic to a fixed smooth elliptic curve; more precisely, if the surface $X$ does not have multiple fibres, then it is the quotient of a complex surface by an infinite cyclic group. The constructions presented here are based on methods used in [Mo] to study bundles over Hopf surfaces. If $X$ is a Hopf surface, then its Picard group is isomorphic to $\mathbb{C}^{*}$ and there exists a universal line bundle on $X \times \mathbb{C}^{*}$; one can then use this bundle to define a natural transformation that takes a locally free sheaf $\mathcal{E}$ on $X$ to a torsion sheaf $\widetilde{\mathcal{L}}$ on $B \times \mathbb{C}^{*}$. Note that the relative Jacobian $J(X)$ of the Hopf surface $X$ is the quotient of $B \times \mathbb{C}^{*}$ by the infinite cyclic group defining the surface; however, the torsion sheaf $\widetilde{\mathcal{L}}$ cannot descend to this quotient. Nonetheless, this problem was solved in [Mo] by constructing a sheaf $\mathcal{N}$ on $B \times \mathbb{C}^{*}$ such that the tensor product $\widetilde{\mathcal{L}} \otimes \mathcal{N}$ descends to $J(X)$. In the sequel, we show that this construction extends to any non-Kähler elliptic fibre bundle; a twisted Fourier-Mukai transform is then defined as the composition of these two operations, taking locally free sheaves on $X$ to torsion sheaves on $J(X)$.

The article is organised as follows. We start by briefly reviewing the existence results that were proven in [BrMo1]. Then in the third section, we define the twisted Fourier-Mukai transform and examine some of its properties. Next, we show that if a vector bundle $E$ is regular on all smooth fibres of $\pi$, then it is completely determined by its spectral cover $S_{E}$ and a certain line bundle on $S_{E}$ (over an elliptic curve, a bundle is said to be regular if its group of automorphisms is of the smallest possible dimension); consequently, we are able to prove that there is a one-to-one correspondence between rank-2 vector bundles with the same smooth spectral cover $S_{E}$ and a finite number of copies of a Prym variety associated to $S_{E}$. We end the article by giving an overview of the methods that can be used to classify bundles that are not regular on at least one fibre of $\pi$.

\section{Holomorphic vector bundles}

Let $X \stackrel{\pi}{\rightarrow} B$ be a minimal non-Kähler elliptic surface, with $B$ a smooth compact connected curve; it is well-known that $X \stackrel{\pi}{\rightarrow} B$ is a quasi-bundle over $B$, that is, all the smooth fibres are pairwise isomorphic and the singular fibres are multiples of elliptic curves [K, Br2]. Let $T$ be the general fibre of $\pi$, which is an elliptic curve, and denote its dual $T^{*}$ (a non-canonical identification $T^{*}:=\operatorname{Pic}^{0}(T) \cong T$ ). In this case, 
the Jacobian surface associated to $X \stackrel{\pi}{\rightarrow} B$ is simply

$$
J(X)=B \times T^{*} \stackrel{p_{1}}{\rightarrow} B
$$

(see, for example, $[\mathrm{K}, \mathrm{BPV}, \mathrm{Br} 1]$ ) and the surface $X$ is obtained from its relative Jacobian by a finite number of logarithmic transformations [K, BPV, BrU].

2.1. Line bundles. Before giving a general description of line bundles on $X$, we begin with torsion line bundles. Suppose that $\pi$ has a multiple fibre $m F$ over the point $b$ in $B$; the line bundle associated to the divisor $F$ of $X$ is then such that $\left(\mathcal{O}_{X}(F)\right)^{m}=\mathcal{O}_{X}(m F)=\pi^{*} \mathcal{O}_{B}(b)$. Let $P_{2}$ denote the subgroup of $\operatorname{Pic}(X)$ generated by $\pi^{*} \operatorname{Pic}(B)$ and the $\mathcal{O}_{X}\left(T_{i}\right)^{\prime}$, where $T_{1}, \ldots, T_{l}$ are the multiple fibres (if any) of $X$. The group of all torsion line bundles on $X$ is then given by

$$
\operatorname{Pic}^{\tau}(X)=P_{2} \otimes \mathbb{C}^{*} .
$$

If $X$ does not have multiple fibres, then the set of all holomorphic line bundles on $X$ with trivial Chern class is given by the zero component of the Picard group $\operatorname{Pic}^{0}(X) \cong$ $\operatorname{Pic}^{0}(B) \times \mathbb{C}^{*}$. In this case, any line bundle in $\operatorname{Pic}^{0}(X)$ is of the form $H \otimes L_{\alpha}$, where $H$ is the pullback to $X$ of an element of $\operatorname{Pic}^{0}(B)$ and $L_{\alpha}$ is the line bundle corresponding to the constant automorphy factor $\alpha \in \mathbb{C}^{*}$; in particular, there exists a universal (Poincaré) line bundle $\mathcal{U}$ on $X \times \operatorname{Pic}^{0}(X)$ whose restriction to $X \times \mathbb{C}^{*}:=X \times\{0\} \times \mathbb{C}^{*}$ is constructed in terms of constant automorphy factors (for details, see [BrMo1]).

For vector bundles on any elliptic surface $X$, restriction to a fibre is a natural operation; however, it is important to note that if $X$ is non-Kähler, then the restriction of any line bundle on $X$ to a smooth fibre of $\pi$ always has degree zero [BrMo1]. Furthermore, even though non-Kähler elliptic surfaces have very few divisors (they are given by the fibres of $\pi$ ), there exist many line bundles on them; we have the following classification [BrMo1].

Proposition 2.2. Let $X \stackrel{\pi}{\rightarrow} B$ be a non-Kähler elliptic surface with general fibre $T$ and $J(X)=B \times T^{*}$ be its relative Jacobian. Fix a section $\Sigma \subset J(X)$. Then:

(i) There exists a line bundle $L$ on $X$ whose restriction to every smooth fibre $T_{b}=\pi^{-1}(b)$ of $\pi$ is the same as the line bundle $\Sigma_{b}$ of degree zero on $T \cong T_{b}$.

(ii) The set of all line bundles on $X$ whose restriction to every smooth fibre of $\pi$ is determined by the section $\Sigma$ is a principal homogeneous space over $P_{2}$.

2.2. The spectral construction. Consider a pair $\left(c_{1}, c_{2}\right) \in N S(X) \times \mathbb{Z}$; then, its discriminant is defined as

$$
\Delta\left(2, c_{1}, c_{2}\right):=\frac{1}{2}\left(c_{2}-\frac{c_{1}^{2}}{4}\right) .
$$

Let $E$ be a rank 2 vector bundle over $X$, with $c_{1}(E)=c_{1}$ and $c_{2}(E)=c_{2}$. For the remainder of the paper, we fix the notation:

$$
\Delta(E):=\Delta\left(2, c_{1}, c_{2}\right) .
$$

To study bundles on $X$, one of our main tools is restriction to the smooth fibres of the fibration $\pi: X \rightarrow B$. Since the restriction of any bundle on $X$ to a fibre $T$ has first Chern class zero, we can consider $E$ as a family of degree zero bundles over the elliptic curve $T$, parametrised by $B$. These families were described in detail in [BrMo1]. For the convenience of the reader, we give a brief summary. 
Given a rank two bundle over $X$, its restriction to a generic fibre of $\pi$ is semistable. More precisely, its restriction to a fibre $\pi^{-1}(b)$ is unstable on at most an isolated set of points $b \in B$; these isolated points are called the jumps of the bundle. Furthermore, there exists a divisor in the relative Jacobian $J(X)=B \times T^{*}$ of $X$, called the spectral curve or cover of the bundle, that encodes the isomorphism class of the bundle over each fibre of $\pi$. The spectral curve can be constructed as follows. If the surface $X$ does not have multiple fibres, then there exists a universal bundle $\mathcal{U}$ on $X \times \operatorname{Pic}^{0}(X)$, whose restriction to $X \times \mathbb{C}^{*}$ is also denoted $\mathcal{U}$; we associate to the rank-2 vector bundle $E$ the sheaf on $B \times \mathbb{C}^{*}$ defined by

$$
\widetilde{\mathcal{L}}:=R^{1} \pi_{*}\left(s^{*} E \otimes \mathcal{U}\right),
$$

where $s: X \times \mathbb{C}^{*} \rightarrow X$ is the projection onto the first factor, $i d$ is the identity map, and $\pi$ also denotes the projection $\pi:=\pi \times i d: X \times \mathbb{C}^{*} \rightarrow B \times \mathbb{C}^{*}$. This sheaf is supported on a divisor $\widetilde{S_{E}}$, defined with multiplicity, that descends to a divisor $S_{E}$ in $J(X)$ of the form

$$
S_{E}:=\left(\sum_{i=1}^{k}\left\{x_{i}\right\} \times T^{*}\right)+\bar{C},
$$

where $\bar{C}$ is a bisection of $J(X)$ and $x_{1}, \cdots, x_{k}$ are points in $B$ that correspond to the jumps of $E$. The spectral curve of $E$ is defined to be the divisor $S_{E}$.

If the fibration $\pi$ has multiple fibres, then one can associate to $X$ a principal $T$-bundle $\pi^{\prime}: X^{\prime} \rightarrow B^{\prime}$ over an $m$-cyclic covering $\varepsilon: B^{\prime} \rightarrow B$, where the integer $m$ depends on the multiplicities of the singular fibres; note that the map $\varepsilon$ induces natural $m$-cyclic coverings $J\left(X^{\prime}\right) \rightarrow J(X)$ and $\psi: X^{\prime} \rightarrow X$. By replacing $X$ with $X^{\prime}$ (which does not have multiple fibres) in the above construction, we obtain the spectral cover $S_{\psi^{*} E}$ of the vector bundle $\psi^{*} E$ as a divisor in $J\left(X^{\prime}\right)$. We then define the spectral cover $S_{E}$ of $E$ to be the projection of $S_{\psi^{*} E}$ in $J(X)$.

Note. The spectral construction can be defined for any rank $r$ vector bundle. In particular, for a line bundle, the spectral cover corresponds to the section of the Jacobian surface $J(X)$ defined in section 2.1.

Remark 2.3. Let $\delta$ be a line bundle on $X$. It then defines an involution $i_{\delta}$ of the relative Jacobian $J(X)=B \times T^{*}$ of $X$, given by $(b, \lambda) \mapsto\left(b, \delta_{b} \otimes \lambda^{-1}\right)$, where $\delta_{b}$ denotes the restriction of $\delta$ to the fibre $T_{b}=\pi^{-1}(b)$. Taking the quotient of $J(X)$ by this involution, we obtain a ruled surface $\mathbb{F}_{\delta}:=J(X) / i_{\delta}$ over $B$. Let $\eta: J(X) \rightarrow \mathbb{F}_{\delta}$ be the canonical map. By construction, the spectral curve $S_{E}$ of a bundle $E$ with determinant $\delta$ is invariant with respect to the involution $i_{\delta}$ and descends to the quotient $\mathbb{F}_{\delta}$; in fact, it is the pullback via $\eta$ of a divisor on $\mathbb{F}_{\delta}$ of the form $\mathcal{G}_{E}:=\sum_{i=1}^{k} f_{i}+A$, where $f_{i}$ is the fibre of the ruled surface $\mathbb{F}_{\delta}$ over the point $x_{i}$ and $A$ is a section of the ruling such that $\eta^{*} A=\bar{C}$.

We have seen that one can associate to every vector bundle on $X$ a spectral cover. Conversely, given a fixed spectral cover without vertical components, there exists at least one rank-2 vector bundle on $X$ associated to it. More precisely, we have the following existence result [BrMo1].

Theorem 2.4. Let $X$ be a non-Kähler elliptic surface and fix a line bundle $\delta$ on $X$. Moreover, let $i_{\delta}$ be the involution of the relative Jacobian $J(X)$ determined by $\delta$ and 
suppose that $\bar{C}$ is a bisection of $J(X)$ that is invariant with respect to $i_{\delta}$. Then, there exists at least one holomorphic rank-2 vector bundle on $X$ that has determinant $\delta$ and spectral cover $\bar{C}$.

Note. Further existence results are given in [BrMo1].

\section{Gerbes and the Fourier-Mukai transform}

For elliptic fibrations with a section, the spectral construction has, more recently, been studied from the point of view of the Fourier-Mukai transform. Let us consider for simplicity a smooth elliptic fibration $Y \stackrel{\pi}{\rightarrow} B$ that has a section but no singular fibres. In this case, the section of the fibration gives a natural identification of $Y$ with its relative Jacobian $J(Y)$, that is, there is a canonical isomorphism $J(Y) \cong Y$ (see, for example, [C1]); moreover, there exists a universal line bundle $\mathcal{P}$ on the fibred product $Y \times_{B} J(Y)=Y \times_{B} Y$. Denote $p_{1}$ and $p_{2}$ the projections of $Y \times_{B} Y$ onto the first and second factors, respectively, and let $E$ be a holomorphic vector bundle on $Y$. As in the previous section, the spectral cover $C$ of $E$ can be described as the support in $Y \cong J(Y)$ of the torsion sheaf $R^{1} p_{2 *}\left(p_{1}^{*} E \otimes \mathcal{P}\right)$, which is the Fourier-Mukai transform of $E$, under some mild assumptions on $E$. Furthermore, if $E$ is semistable of degree zero on the generic fibre of $\pi$, then the restriction of $R^{1} p_{2 *}\left(p_{1}^{*} E \otimes \mathcal{P}\right)$ to $C$ is a rank one coherent torsion free sheaf $L$ and one can recover the bundle $E$ from the spectral data $(C, L)$.

Let us turn to the case of a non-Kähler elliptic fibre bundle $X \stackrel{\pi}{\rightarrow} B$; given the non-Kähler condition, $\pi$ is now an elliptic fibration without a section - a genus one fibration in the terminology of $[\mathrm{DP}]$. Note that $X$ is not isomorphic to its relative Jacobian; indeed, recall that if the general fibre of $X$ is the elliptic curve $T$, then $J(X)=B \times T^{*}$. In addition, there is no universal line bundle on $X \times_{B} J(X)$. To construct spectral covers of vector bundles on $X$, we bypassed this problem by working instead with the universal line bundle $\mathcal{U}$ that exists on $X \times \mathbb{C}^{*}$ (see sections 2.1 and 2.2 ). Consider a rank- 2 vector bundle $E$ on $X$. In this section, we show that the spectral cover $S_{E} \subset J(X)$ of $E$ can also be determined as the support of a torsion sheaf $\mathcal{L}$ on $J(X)$, leading us to the natural definition of a twisted Fourier-Mukai transform $\Phi$ for locally free sheaves on $X$; in particular, $\Phi(E)=\mathcal{L}$. Some properties of this transform are then examined.

3.1. Gerbes. Let $X$ be a general genus one fibration without singular fibres. Then, as in the case of non-Kähler elliptic surfaces, $X$ is not isomorphic to its relative Jacobian $J(X)$ and distinct fibrations may have the same relative Jacobian; the information lost by passing from $X$ to its Jacobian is, however, encoded in the class of $X$ in the Tate-Shavarevich group of $J(X)$, or equivalently, by an $\mathcal{O}_{J(X)}^{*}$-gerbe $\xi$ on $J(X)$. Furthermore, even though there is no universal sheaf on $X \times_{B} J(X)$, universal $\xi$-twisted sheaves do exist [C1]. An equivalence between derived categories of twisted sheaves, called a "gerby" Fourier-Mukai transform, can then be constructed by using these universal twisted sheaves (see [DP] for precise statements).

For example, if $X$ is a non-Kähler principal $T$-bundle over the curve $B$, then the $\mathcal{O}_{J(X)}^{*}$-gerbe corresponding to $X$ is given by a cohomology class $\xi \in H^{1}\left(B, \mathcal{O}_{B}(T)\right)$, where $\mathcal{O}_{B}(T)$ denotes the sheaf of germs of locally holomorphic maps from $B$ to $T$, and there exists a $\xi$-twisted universal sheaf $\mathcal{U}_{\xi}$ on $X \times_{B} J(X)=X \times T^{*}$. In this case, 
the results of $[\mathrm{DP}]$ state that the twisted Fourier-Mukai transform defined by $\mathcal{U}_{\xi}$ is an equivalence between the derived category $D^{b}(J(X), \xi)$ of $\xi$-twisted sheaves on $J(X)$ and the derived category $D^{b}(X)$ of sheaves on $X$. But in this paper, we use the gerbe $\xi$ to construct an explicit twist of the torsion sheaf $\widetilde{\mathcal{L}}:=R^{1} \pi_{*}\left(s^{*} E \otimes \mathcal{U}\right)$, on $B \times \mathbb{C}^{*}$, that descends to $J(X)=B \times T^{*}$.

The cohomology class $\xi$ induces an isomorphism of the surface $X$ with a quotient of the form

$$
X=\Theta^{*} /\langle\tau\rangle
$$

where $\Theta$ is a line bundle on $B$ with positive Chern class $d, \Theta^{*}$ is the complement of the zero section in the total space of $\Theta$, and $\langle\tau\rangle$ is the multiplicative cyclic group generated by a fixed complex number $\tau \in \mathbb{C}$, with $|\tau|>1$; given this identification, every fibre of $\pi$ is isomorphic to the elliptic curve $\mathbb{C}^{*} / \tau^{n}$. Hence, multiplication by $\tau$ defines a natural $\mathbb{Z}$-action on $X \times \mathbb{C}^{*}$ that is trivial on $X$, inducing the quotient $\left(X \times \mathbb{C}^{*}\right) / \mathbb{Z}=X \times T^{*}=X \times_{B} J(X)$.

We saw in section 2.1 that every torsion line bundle $L \in \operatorname{Pic}^{0}(X)$ decomposes uniquely as $L=H \otimes L_{\alpha}$, for $H \in \pi^{*} \operatorname{Pic}^{0}(B)$ and $\alpha \in \mathbb{C}^{*}$. However, if one considers the constant factor of automorphy $\tau \in \mathbb{C}^{*}$, then one easily verifies that $L_{\tau}$ is in fact the pullback of a line bundle on $B$ of degree $-d$, also denoted $L_{\tau}$. Note that there is also a natural $\mathbb{Z}$-action on $B \times \mathbb{C}^{*}$, defined as multiplication by $\tau$ on the second factor, and $\left(B \times \mathbb{C}^{*}\right) / \mathbb{Z} \cong J(X)$. Moreover, this action extends to the torsion sheaf $\widetilde{\mathcal{L}}:=R^{1} \pi_{*}\left(s^{*} E \otimes \mathcal{U}\right)$, taking the stalk $\widetilde{\mathcal{L}}_{(x, \alpha)}$ to $\widetilde{\mathcal{L}}_{(x, \tau \alpha)} \otimes L_{\tau^{-1}, x}$ [BrMo1]. Therefore, $\widetilde{\mathcal{L}}$ cannot descend to $J(X)$ because it is not invariant with respect to this action. To fix this problem, we construct a sheaf $\mathcal{N}$ on $B \times \mathbb{C}^{*}$ and a $\mathbb{Z}$-action that leaves the tensor product $\widetilde{\mathcal{L}} \otimes \mathcal{N}$ invariant.

Let us choose a point $b_{0}$ in $B$ over which the graph of $E$ does not have a vertical component; without loss of generality, we assume that the line bundle $L_{\tau}$ is given by the divisor $-d b_{0}$ (if the divisor associated to $L_{\tau}$ is instead $\sum_{i} b_{i}-\sum_{j} b_{j}$, the construction below follows verbatim, but the notation is more complicated). Let $W=$ $\left(b_{0} \times \mathbb{C}^{*}\right) \cap \widetilde{S_{E}}$ be the set of points on $\widetilde{S_{E}}$ lying above $b_{0}$. If $(a, b)$ is a representation of the pair of points on $S_{E}$ above $b_{0}$, then $W$ is the set of all translates of this pair by $\tau$, that is, $W=\bigcup_{i \in \mathbb{Z}}\left(\tau^{i} a, \tau^{i} b\right)$. Also, $(a+b)$ is a divisor on $\widetilde{S_{E}}$ and we denote $T^{i}(a+b):=\tau^{i} a+\tau^{i} b$ the translate of $(a+b)$ by $\tau^{i}$; we define a divisor on $\widetilde{S_{E}}$ as the locally finite sum

$$
D_{E, b_{0}}:=\sum_{i \in \mathbb{Z}} i d T^{i}(a+b)
$$

Let $\mathcal{N}$ be the line bundle on $\widetilde{S_{E}}$ associated to the invertible sheaf $\mathcal{O}_{\widetilde{S_{E}}}\left(D_{E, b_{0}}\right)$; we also denote by $\mathcal{N}$ the line bundle considered as a sheaf on $B \times \mathbb{C}^{*}$. 
Fix a section $\gamma$ of the line bundle $L_{\tau}$; we use it to define the following $\mathbb{Z}$-action on the sheaf $\widetilde{\mathcal{L}} \otimes \mathcal{N}$ over $B \times \mathbb{C}^{*}$ :

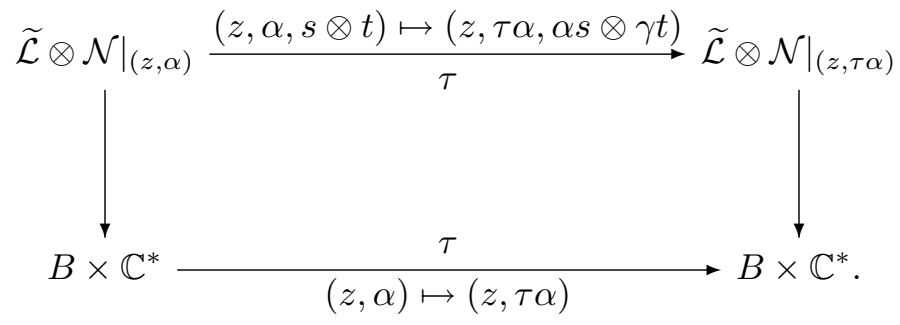

Clearly, the sheaf $\widetilde{\mathcal{L}} \otimes \mathcal{N}$ is invariant under this action and descends to the quotient $J(X)$; we denote the quotient sheaf

$$
\mathcal{L}:=(\widetilde{\mathcal{L}} \otimes \mathcal{N}) / \sim .
$$

Note that the support of $\mathcal{L}$ is $S_{E}$; moreover, if we take the pull back of $\mathcal{L}$ to $B \times \mathbb{C}^{*}$ and tensor it by $\mathcal{N}^{*}$, then we recover $\widetilde{\mathcal{L}}$ (as above, we also denote $\mathcal{N}^{*}$ the sheaf on $B \times \mathbb{C}^{*}$ obtained by extending the line bundle $\mathcal{N}^{*}$ on $\widetilde{S_{E}}$ by zero outside $\widetilde{S_{E}}$ ).

3.2. Properties of the Fourier-Mukai transform. Consider the following commutative diagram:

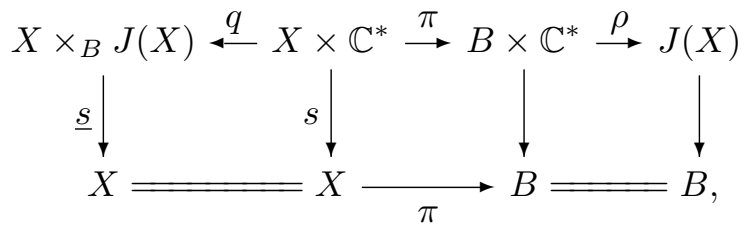

where $\underline{s}$ is projection onto the first factor, $q: X \times \mathbb{C}^{*} \rightarrow X \times T^{*}=X \times{ }_{B} J(X)$ and $\rho: B \times \mathbb{C}^{*} \rightarrow B \times T^{*}=J(X)$ are the natural quotient maps induced by the $\mathbb{Z}$-actions defined in section 3.1, and $\pi$ and $s$ are the projections defined in section 2.2. Given a locally free sheaf $E$ on $X$, we define the twisted Fourier-Mukai transform to be the complex of sheaves $\Phi(E)$ on $J(X)$ given by

$$
\Phi(E):=\left(R \pi_{*}\left(s^{*} E \otimes \mathcal{U}\right) \otimes \mathcal{N}\right) / \sim .
$$

Conversely, if $\mathcal{L}$ is a sheaf on $J(X)$, we define the "inverse" twisted Fourier-Mukai transform as the complex of sheaves $\hat{\Phi}(\mathcal{L})$ on $X$ given by

$$
\hat{\Phi}(\mathcal{L}):=R \underline{s}_{*}\left(\left(\pi^{*}\left(\left(\rho^{*} \mathcal{L}\right) \otimes \mathcal{N}^{*}\right) \otimes \mathcal{U}^{*}\right) / \sim\right) .
$$

Referring to section 3.1, the sheaves of these complexes are well-defined; however, we should point out that we use the term "inverse" only in the sense that the transforms $\Phi$ and $\hat{\Phi}$ are inverses of each other on certain classes of sheaves, as will become clear in the following. We state some of their properties in:

Theorem 3.1. (i) Suppose that $E$ is a rank-2 vector bundle on $X$ without jumps. Then, $\Phi^{0}(E)=0$ and $\hat{\Phi}^{0}\left(\Phi^{1}(E)\right)=E$.

(ii) If $\mathcal{L}$ is a torsion sheaf on $J(X)$, supported on a bisection $C \subset J(X)$, that has rank 1 on the smooth points of $C$ and rank at most 2 on the singular ones, then $\hat{\Phi}^{1}(\mathcal{L})=0$ and $\Phi^{1}\left(\hat{\Phi}^{0}(\mathcal{L})\right)=\mathcal{L}$. 
Proof. Let $E$ be a rank-2 vector bundle on $X$ without jumps. If one unravels the definitions, then one sees that

$$
\hat{\Phi}^{0}\left(\Phi^{1}(E)\right)=\underline{s}_{*}\left(\left(\left(\pi^{*} R^{1} \pi_{*}\left(s^{*} E \otimes \mathcal{U}\right)\right) \otimes \mathcal{U}^{*}\right) / \sim\right) ;
$$

moreover, flat base change induces a natural isomorphism $\pi^{*} R^{1} \pi_{*}(\mathcal{F})=\mathcal{F}$ for any coherent sheaf $\mathcal{F}$ on $B \times \mathbb{C}^{*}$, implying that $\hat{\Phi}^{0}\left(\Phi^{1}(E)\right)=\underline{s}_{*}\left(s^{*} E / \sim\right)$. However, since $E$ is invariant under the $\mathbb{Z}$-action, we have $s^{*} E / \sim=\underline{s}^{*} E$ and $\hat{\Phi}^{0}\left(\Phi^{1}(E)\right)=\underline{s}_{*}\left(\underline{s}^{*} E\right) \cong$ $E$, proving (i).

Note that for any coherent sheaf $\mathcal{G}$ on $X \times_{B} J(X)$, there is an identification $s^{*} \underline{s}_{*}(\mathcal{G})=q^{*}(\mathcal{G})$, which is again due to flat base change. Consequently, if $\mathcal{L}$ is a torsion sheaf on $J(X)$ satisfying the hypothesis of part (ii), we have

$$
\Phi^{1}\left(\hat{\Phi}^{0}(\mathcal{L})\right)=\left(R^{1} \pi_{*}\left(\pi^{*}\left(\rho^{*} \mathcal{L} \otimes \mathcal{N}^{*}\right)\right) \otimes \mathcal{N}\right) / \sim .
$$

There is a natural map $\rho^{*} \mathcal{L} \otimes \mathcal{N}^{*} \rightarrow R^{1} \pi_{*}\left(\pi^{*}\left(\rho^{*} \mathcal{L} \otimes \mathcal{N}^{*}\right)\right)$; since both sheaves are supported on $\rho^{*} C$ and have naturally isomorphic fibres, this map must be an isomorphism, giving $\Phi^{1}\left(\hat{\Phi}^{0}(\mathcal{L})\right)=\left(\left(\rho^{*} \mathcal{L} \otimes \mathcal{N}^{*}\right) \otimes \mathcal{N}\right) / \sim=\mathcal{L}$.

Remark 3.2. Suppose that the bisection $C$ is smooth. If the torsion sheaf $\mathcal{L}$ satisfies the conditions of Theorem 3.1 (ii), then its restriction to $C$ is a line bundle. Denote $L:=\left(\pi^{*}\left(\left(\rho^{*} \mathcal{L}\right) \otimes \mathcal{N}^{*}\right) \otimes \mathcal{U}^{*}\right) / \sim$ the quotient sheaf on $X \times_{B} J(X)$; one easily verifies that the support of $L$ is the principal $T$-bundle $W:=X \times{ }_{B} C$ and that the restriction of $L$ to $W$ is a line bundle. Let $\tilde{\gamma}: W \rightarrow X$ be the natural projection. We then have the following identification:

$$
\hat{\Phi}^{0}(\mathcal{L})=\underline{s}_{*}(L)=\tilde{\gamma}_{*}\left(\left.L\right|_{W}\right) .
$$

In particular, we see that if $E$ is a rank-2 vector bundle with spectral cover $C$ and $\Phi^{1}(E)=\mathcal{L}$, then $L_{W}:=\left.L\right|_{W}$ is the unique line bundle on $W$ such that $\tilde{\gamma}_{*}\left(L_{W}\right)=E$.

\section{Regular rank two vector bundles}

4.1. Definition and existence. Over a smooth elliptic curve, a rank-2 vector bundle of degree zero is said to be regular if its group of automorphisms is of the smallest dimension, that is, it is a semistale bundle that is never isomorphic to $\lambda_{0} \oplus \lambda_{0}$, with $\lambda_{0} \in \mathrm{Pic}^{0}(T)$. If $E$ is a holomorphic rank-2 vector bundle on an elliptic surface $X$, then $E$ is regular if and only if its restriction to a fibre $T$ is always regular. One can easily show that if a rank-2 vector bundle has a smooth spectral cover, then it is regular (see, for example, [FMW, Mo]); given Theorem 2.4, regular bundles therefore always exits. However, regular bundles do not always have smooth spectral covers, as stated in:

Proposition 4.1. Let $X$ be a non-Kähler elliptic fibre bundle over the curve $B$ and let $\delta$ be a line bundle on $X$. Consider a (not necessarily smooth) bisection $C$ of the relative Jacobian $J(X)$ of $X$ that is invariant with respect to the involution $i_{\delta}$ of $J(X)$ determined by $\delta$. Then, there exists at least one regular bundle on $X$ with determinant $\delta$ and spectral cover $C$.

Proof. Let us consider the section $A$ of $\mathbb{F}_{\delta}$ corresponding to $C$ (see remark 2.3). Locally, over an open disc $D$ of $B$, this section is the graph of a rational map $\varrho$ : $D \rightarrow \mathbb{P}^{1}$. Choose a line bundle $\lambda$ over $T$ with $c_{1}(\lambda)=-1$; non-trivial extensions of $\lambda^{*}$ 
by $\lambda$ are therefore regular rank- 2 vector bundles over $T$, which are parametrised by $\mathbb{P}\left(H^{1}\left(T, \lambda^{2}\right)\right) \cong \mathbb{P}^{1}$. If we write $\varrho$ as a quotient $p(b) / q(b)$, where $p$ and $q$ are coprime polynomials, we can define a regular bundle on $D \times T$, whose graph is the restriction of $A$ to $D$, by the extension

$$
\begin{aligned}
D & \longrightarrow H^{1}\left(T, \lambda^{2}\right), \\
b & \longmapsto(p(b), q(b)) .
\end{aligned}
$$

Moreover, if two regular bundles on $D \times T$ have the same graph, given by a rational map $\varrho: D \rightarrow \mathbb{P}^{1}$, then they are isomorphic (see Lemma 5.1.2 of $[\mathrm{BH}]$ ); this is also true for any simply connected subset $D$ of $B$, in which case the isomorphism can be chosen to be an $S L(2, \mathbb{C})$-isomorphism. Let $\left\{D_{i}\right\}$ be an open cover of $B$ such that every open set $D_{i}$ is a disc in $B$ and the intersection of any two open sets is simply connected. The extension (4.2) gives regular bundles over each $D_{i}$, which can then be glued together to give a regular bundle on $X$ with the required properties.

4.2. Classification of regular bundles. Assume that the surface $X$ has multiple fibres $m_{1} T_{1}, \ldots, m_{l} T_{l}$ over the points $b_{1}, \ldots, b_{l}$ in $B$. We fix a line bundle $\delta$ on $X$ and its associated involution $i:=i_{\delta}$ of the Jacobian surface $J(X)$. Consider a smooth irreducible bisection $C$ of $J(X)=B \times T^{*}$, invariant under the involution $i$, whose induced double cover $\gamma: C \rightarrow B$ of $B$ is branched. Let $W$ be the normalisation of $X \times{ }_{B} C$. As $C$ is a double cover of $B$, then $W$ is also a double cover of $X$ and we have the following commutative diagram

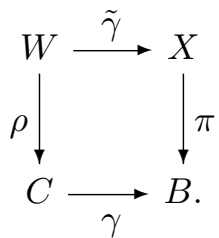

Note that, to each multiple fibre $m_{i} T_{i}$ of $\pi$, there corresponds a pair of multiple fibres $m_{i} T_{i}^{\prime}$ and $m_{i} T_{i}^{\prime \prime}$ of $\rho$ (that both get mapped to $m_{i} T_{i}$ under $\tilde{\gamma}$ ); furthermore, if the fibre $m_{j} T_{j}$ lies over a branch point of $C \stackrel{\gamma}{\rightarrow} B$, then $m_{j} T_{j}^{\prime}=m_{j} T_{j}^{\prime \prime}$.

Let $P_{2, W}$ be the subgroup of $\operatorname{Pic}(\mathrm{W})$ generated by $\rho^{*} \operatorname{Pic}(\mathrm{C}), \mathcal{O}_{W}\left(T_{i}^{\prime}\right)$, and $\mathcal{O}_{W}\left(T_{i}^{\prime \prime}\right)$, $i=1, \ldots, l$; we define the following subgroup of $P_{2, W}$ :

$$
P_{2, W}^{0}:=\left\{\lambda \in P_{2, W}: \tilde{\gamma}_{*}\left(c_{1}(\lambda)\right)=0 \text { in } H^{2}(X, \mathbb{Z})\right\} .
$$

Finally, suppose that there are $s$ multiple fibres $m_{i_{1}} T_{i_{1}}, \ldots, m_{i_{s}} T_{i_{s}}$ of $\pi$ that do not lie over branch points of $C \stackrel{\gamma}{\rightarrow} B$. Then, we denote

$$
P_{2, W}^{T^{\prime}-T^{\prime \prime}}:=\left\langle\mathcal{O}_{W}\left(T_{i_{j}}^{\prime}-T_{i_{j}}^{\prime \prime}\right), j=1, \ldots, s\right\rangle \subset P_{2, W}
$$

the finite subgroup of order $m_{i_{1}} \ldots m_{i_{s}}$ generated by line bundles on $W$ of the form $\mathcal{O}_{W}\left(T_{i_{j}}^{\prime}-T_{i_{j}}^{\prime \prime}\right)$. Given the above notation, we can now state the main result of this section.

Theorem 4.5. Let us denote $\Sigma_{C}$ the section of $J(W)=C \times T^{*}$ that corresponds to the map $C \rightarrow J(X)$. Fix a line bundle $L$ on $W$ that corresponds to the section $\Sigma_{C}$ and is such that $\operatorname{det}\left(\tilde{\gamma}_{*}(L)\right)=\delta$. All rank-2 vector bundles on $X$ with determinant $\delta$ and spectral cover $C$ are then given precisely by

$$
\tilde{\gamma}_{*}(L \otimes \lambda), \quad \lambda \in P_{2, W, \bar{\iota}}^{0},
$$


where $\bar{\iota}$ denotes the involution on $W$ that interchanges the sheets of $\tilde{\gamma}$ and

$$
P_{2, W, \bar{\iota}}^{0}:=\left\{\lambda \in P_{2, W}^{0} \mid \iota^{*} \lambda \otimes \lambda=\mathcal{O}_{W}\right\} .
$$

Moreover, there is a natural exact sequence

$$
0 \rightarrow \operatorname{Prym}(C / B) \rightarrow P_{2, W, \bar{\iota}}^{0} \rightarrow P_{2, W}^{T^{\prime}-T^{\prime \prime}} \rightarrow 0,
$$

where Prym $(C / B)$ is the Prym variety associated to the (branched) double cover $C \stackrel{\gamma}{\rightarrow} B$. Note that if $X$ does not have multiple fibres, then $P_{2, W, \bar{\imath}}^{0} \cong \operatorname{Prym}(C / B)$.

Remark. If one does not fix the determinant, rank-2 vector bundles on $X$ with first Chern class $c_{1}(\delta)$ and spectral cover $C$ are then parametrised by the group $P_{2, W}^{0}$, which is isomorphic to $m_{i_{1}} m_{i_{2}} \ldots m_{i_{s}}$ copies of the Jacobian $J(C)$ of $C$.

Proof. Consider a rank-2 vector bundles $E$ with determinant $\delta$ and spectral cover $C$. Referring to remark 3.2, if $X$ does not have multiple fibres, we can associate to $E$ a unique line bundle $L$ on $W$ such that $E=\tilde{\gamma}_{*}(L)$. If $X$ has multiple fibres, the same can be said of the pullback $\psi^{*} E$ of $E$ to $X^{\prime}$, where $X^{\prime}$ is the $m$-to-one cover of $X$ (without multiple fibres) described in section 2.2. Indeed, if $N_{1}$ and $N_{2}$ are non-isomorphic line bundles on $W$, they must generate non-isomorphic rank-2 vector bundles on $X$, otherwise, their pullbacks to $X^{\prime}$ would contradict the above. In other words, whether $X$ has multiple fibres or not, there is a one-to-one correspondence between rank-2 vector bundles, with determinant $\delta$ and spectral cover $C$, and line bundles $N$ on $W$, associated to the section $\Sigma_{C} \subset J(W)$, such that $\operatorname{det}\left(\tilde{\gamma}_{*}(N)\right)=\delta$.

Fix a line bundle $L$ on $W$ that corresponds to the section $\Sigma_{C}$ and is such that $\operatorname{det}\left(\tilde{\gamma}_{*}(L)\right)=\delta$. Recall that any line bundle corresponding to $\Sigma_{C}$ is of the form $L \otimes \lambda$, where $\lambda$ is an element of $P_{2, W}$. Hence, consider a line bundle $L^{\prime}=L \otimes \lambda, \lambda \in P_{2, W}$, such that $\operatorname{det}\left(\tilde{\gamma}_{*}\left(L^{\prime}\right)\right)=\delta$; in particular, $c_{1}\left(\tilde{\gamma}_{*}\left(L^{\prime}\right)\right)=c_{1}(\delta)$. Since

$$
c_{1}\left(\tilde{\gamma}_{*}(L \otimes \lambda)\right)=c_{1}\left(\tilde{\gamma}_{*}(L)\right)+\tilde{\gamma}_{*}\left(c_{1}(\lambda)\right),
$$

we see that $c_{1}\left(\tilde{\gamma}_{*}\left(L^{\prime}\right)\right)=c_{1}(\delta)$ if and only if $\tilde{\gamma}_{*}\left(c_{1}(\lambda)\right)=0$, implying that $\lambda \in P_{2, W}^{0}$. For any line bundle $N$ on $W$, there is an exact sequence on $W$

$$
0 \rightarrow \tilde{\iota}^{*} N \otimes \tilde{\gamma}^{*} L_{0}^{-1} \rightarrow \tilde{\gamma}^{*} \tilde{\gamma}_{*}(N) \rightarrow N \rightarrow 0,
$$

where $L_{0}$ is a line bundle on $X$ such that $L_{0}^{2}=\mathcal{O}_{X}(G)$ and $G \subset X$ is the branch divisor of the double cover $\tilde{\gamma}: W \rightarrow X$ (see Lemma 29, Chapter 2, in [F]). Inserting the line bundles $L$ and $L^{\prime}$ in the exact sequence (4.6), we have

$$
\operatorname{det}\left(\tilde{\gamma}^{*} \tilde{\gamma}_{*}\left(L^{\prime}\right)\right)=\operatorname{det}\left(\tilde{\gamma}^{*} \tilde{\gamma}_{*}(L)\right) \otimes\left(\bar{\iota}^{*} \lambda \otimes \lambda\right) .
$$

Consequently, $\lambda$ must satisfy the equation

$$
\bar{\iota}^{*} \lambda \otimes \lambda=\mathcal{O}_{W} .
$$

The set of all rank- 2 vector bundles on $X$ with determinant $\delta$ and spectral cover $C$ is therefore parametrised by $P_{2, W, \bar{\imath}}^{0}$.

We end by determining the generators of the group $P_{2, W, \bar{\iota}}^{0}$. Let $\lambda \in P_{2, W}^{0}$. One easily verifies that if $\lambda \in \rho^{*} \operatorname{Pic}(\mathrm{C})$, then $\tilde{\gamma}_{*}\left(c_{1}(\lambda)\right)=0$ if and only if $\lambda \in \rho^{*} \operatorname{Pic}^{0}(\mathrm{C})$. We then assume that the divisor of the line bundle $\lambda$ contains multiple fibres of $W \stackrel{\rho}{\rightarrow} C$. Clearly, if it contains the multiple $a_{i} T_{i}^{\prime}$, with $0 \leq a_{i} \leq m_{i}-1$, then $\tilde{\gamma}_{*}\left(c_{1}(\lambda)\right)=0$ if and only if it also contains $a_{i} T_{i}^{\prime \prime}$. Hence, the divisor of $\lambda$ must contain multiples of the form $a_{1}\left(T_{1}^{\prime}-T_{1}^{\prime \prime}\right)+\cdots+a_{r}\left(T_{l}^{\prime}-T_{l}^{\prime \prime}\right)$, with $0 \leq a_{i} \leq m_{i}-1$, for all $i=1, \ldots, l$. Since 
$T_{j}^{\prime}=T_{j}^{\prime \prime}$ whenever $T_{j}$ lies over a branch point of $\gamma$, we see that $P_{2, W}^{0}$ is generated by the elements of $\rho^{*} \operatorname{Pic}^{0}(\mathrm{C})$ and $P_{2, W}^{T^{\prime}-T^{\prime \prime}}$. Note that every element of $P_{2, W}^{T^{\prime}-T^{\prime \prime}}$ satisfies equation (4.7). Moreover, given that the double cover $C \stackrel{\gamma}{\rightarrow} B$ is ramified, we have

$$
\left\{\lambda \in \operatorname{Pic}^{0}(\mathrm{C}): \iota^{*} \lambda \otimes \lambda=\mathcal{O}_{\mathrm{C}}\right\} \cong \operatorname{ker}\left(\gamma_{*}\right) \cong \operatorname{Prym}(\mathrm{C} / \mathrm{B})
$$

where $\gamma_{*}$ is the norm map associated to $\gamma$ (see [Mum], sections 2 and 3); thus, since $\rho^{*}: \operatorname{Pic}(\mathrm{B}) \rightarrow \operatorname{Pic}(\mathrm{C})$ is injective, the elements of $\rho^{*} \operatorname{Pic}^{0}(\mathrm{C})$ satisfying (4.7) are identified with $\operatorname{Prym}(C / B)$.

\section{The local geometry of a jump}

We finish this paper with some remarks concerning rank-2 vector bundles on $X$ with jumps. We begin by fixing some notation. Let $E$ be such a bundle and assume, for simplicity, that it has a single jump over the smooth fibre $T=\pi^{-1}\left(x_{0}\right)$. The multiplicity $\mu$ of the jump is then less than or equal to the non-negative integer $n_{E}:=-c h_{2}(E)[\mathrm{BrMo1}]$ and the spectral cover of the bundle has two components:

$$
S_{E}=\mu\left(\left\{x_{0}\right\} \times T^{*}\right)+\bar{C},
$$

where $\bar{C}$ is a bisection of $J(X)$ whose degree over $T^{*}$ is equal to $n_{E}-\mu$. Finally, if $\delta$ is the determinant of $E$, the restriction of $E$ to the fibre $T$ is then of the form $\lambda \oplus\left(\lambda^{*} \otimes \delta_{x_{0}}\right)$, for some $\lambda \in \mathrm{Pic}^{-h}(T), h>0$. The integer $h$ is called the height of the jump at $T$; the height and multiplicity satisfy the inequality $h \leq \mu$.

Suppose that $X$ has multiple fibres and consider one of them, say $m_{0} T_{0}$. As in section 2.2, one can associate to $X$ an elliptic quasi-bundle $\pi^{\prime}: X^{\prime} \rightarrow B^{\prime}$, over an $m_{0}$-cyclic covering $\varepsilon: B^{\prime} \rightarrow B$, such that $T_{0}^{\prime}:=\psi^{-1}\left(T_{0}\right) \subset X^{\prime}$ is a smooth fibre of $\pi^{\prime}$, where $\psi: X^{\prime} \rightarrow X$ is the $m_{0}$-cyclic covering induced by $\varepsilon$. We then say that $E$ has a jump over $T_{0}$ if and only if the restriction of $\psi^{*} E$ to the fibre $T_{0}^{\prime}$ is unstable. Consequently, the height and multiplicity of the jump of $E$ over $T_{0}$ are defined as the height and multiplicity of the jump of $\psi^{*} E$ over $T_{0}^{\prime}$.

5.1. The jumping sequence of a vector bundle. A key ingredient in the description of a jump is the notion of elementary modification of a vector bundle; for its basic properties, we refer the reader to $[\mathrm{F}]$. Consider a rank- 2 vector bundle $E$ on $X$ with $\operatorname{det}(E)=\delta, c_{2}(E)=c_{2}$, and spectral cover $S_{E}=\left(\sum_{i=1}^{k}\left\{x_{i}\right\} \times T^{*}\right)+\bar{C}$. If $N$ is a line bundle on the smooth fibre $T_{0}:=\pi^{-1}\left(x_{0}\right)$ for which there exists at least one surjection $\left.E\right|_{T_{0}} \rightarrow N$, then any elementary modification $V$ of $E$ determined by $N$ has invariants $\operatorname{det}(V)=\delta\left(-T_{0}\right), c_{2}(V)=c_{2}+\operatorname{deg}(N)$, and spectral cover

$$
S_{V}=\operatorname{deg}(N)\left(\left\{x_{0}\right\} \times T^{*}\right)+\left(\sum_{i=1}^{k}\left\{x_{i}\right\} \times T^{*}\right)+\bar{C} .
$$

Note that the restriction of $V$ to $T_{0}$ is of the form $N \oplus\left(N^{*} \otimes \delta_{x_{0}}\right)$.

Suppose that $E$ is unstable on the fibre $T$, splitting as $\left.E\right|_{T}=\lambda \oplus\left(\lambda^{*} \otimes \delta_{x_{0}}\right)$ for some $\lambda \in \operatorname{Pic}^{-h}(T), h>0$. Then, up to a multiple of the identity, there is a unique surjection $\left.E\right|_{T} \rightarrow \lambda$, which defines a canonical elementary modification of $E$ that we denote $\bar{E}$. This elementary modification is called allowable $[\mathrm{F}]$. Consequently, we can associate to $E$ a finite sequence $\left\{\bar{E}_{1}, \bar{E}_{2}, \ldots, \bar{E}_{l}\right\}$ of allowable elementary modifications 
such that $\bar{E}_{l}$ is the only element of the sequence that does not have a jump at $T$. This sequence induces the following important invariants of a jump.

Definition 5.1. Let $T$ be a smooth fibre of $\pi$. Suppose that the vector bundle $E$ has a jump over $T$ and consider the corresponding sequence of allowable elementary modifications $\left\{\bar{E}_{1}, \bar{E}_{2}, \ldots, \bar{E}_{l}\right\}$. The integer $l$ is called the length of the jump at $T$. The jumping sequence of $T$ is defined as the set of integers $\left\{h_{0}, h_{1}, \ldots, h_{l-1}\right\}$, where $h_{0}=h$ is the height of $E$ and $h_{i}$ is the height of $\bar{E}_{i}$, for $0<i \leq l-1$.

If the vector bundle $E$ has a jump over a multiple fibre $m_{0} T_{0}$ of $\pi$, we define the length and jumping sequence of $T_{0}$ to be the length and jumping sequence of the jump of $\psi^{*} E$ over the smooth fibre $T_{0}^{\prime}=\psi^{-1}\left(T_{0}\right)$ of $\psi$, where $\psi: X^{\prime} \rightarrow X$ is the $m_{0}$-cyclic covering defined at the beginning this section.

Proposition 5.2. Let $E$ be a rank-2 vector bundle on a non-Kähler elliptic surface that has a jump of multiplicity $\mu$ at $T$ with jumping sequence $\left\{h_{0}, h_{1}, \ldots, h_{l-1}\right\}$. Then,

(i) For all $0 \leq k \leq l-1$, we have $h_{k+1} \leq h_{k}$.

(ii) Its allowable elementary modification $\bar{E}$ has a jump of length $l-1$ over $T$ with jumping sequence $\left\{h_{1}, \ldots, h_{l-1}\right\}$.

(iii) The multiplicity of the jump is the sum of the jumping sequence, that is, $\mu=\sum_{i=0}^{l-1} h_{i}$.

(iv) An elementary modification $\widetilde{E}$ of $E$ determined by a line bundle of degree $r \geq h_{0}$ has a jump of length $l+1$ over $T$ with jumping sequence $\left\{r, h_{0}, h_{1}, \ldots, h_{l-1}\right\}$. Moreover, the allowable elementary modification of $\widetilde{E}$ is $E$.

5.2. Generic jumps. If one starts with a vector bundle $E$ that is semistable on every fibre of $\pi$, then one can introduce jumps over the smooth fibres of $\pi$ by performing elementary modifications; however, this implies the existence of a surjection from $E$ to a positive line bundle $N$ over such a fibre $T$. If the bundle $E$ is regular, then such a surjection always exists. But if the bundle $E$ is not regular on the fibre $T$, these surjections fail to exist precisely for line bundles $N$ of degree one; in this case, one cannot add a jump of multiplicity one to $E$ over the fibre $T$.

Let us fix a spectral cover $S=\left(\sum_{i=1}^{k}\left\{x_{i}\right\} \times T^{*}\right)+\bar{C}$ such that $\pi^{-1}\left(x_{i}\right)$ is a smooth fibre, for all $i=1, \ldots, k$. Suppose that the bisection $\bar{C}$ is invariant with respect to an involution of $J(X)$ determined by a line bundle $\delta$ on $X$. The generic bundle with spectral cover $S$ can be constructed as follows. Start with a bundle $E_{0}$ that has determinant $\delta(k T)$ and spectral cover $\bar{C}$, which is regular on the fibres $\pi^{-1}\left(x_{i}\right)$. If the surface $X$ does not have multiple fibres, then this is always possible (see Proposition 4.1). Fix a line bundle $N$ on $T$ of degree one. Finally, perform $k$ elementary modifications with respect to $N$ on the fibres $\pi^{-1}\left(x_{i}\right)$, counting multiplicity, to obtain a vector bundle whose jumps have the correct multiplicities. In this case, every jump has jumping sequence $\{1, \ldots, 1\}$. This construction is, however, far from canonical because it depends on the following: a choice of rank- 2 vector bundle $E_{0}$, a choice of line bundle $N$ of degree one and surjections to $N$.

In general, we can assign to a jump $\pi^{-1}\left(x_{i}\right)$ any jumping sequence $\left\{h_{0}, \ldots, h_{l-1}\right\}$, as long as $\sum_{i=1}^{l-1} h_{i}$ is equal to the multiplicity of the vertical component $\left\{x_{i}\right\} \times T^{*}$ in $S$, in which case we will have to choose a different line bundle $N$ 
for each distinct integer in $\left\{h_{0}, \ldots, h_{l-1}\right\}$. For a detailed classification of such jumps, we refer the reader to [Mo, BrMo2].

\section{Acknowledgements.}

The first author would like to express his gratitude to the Max Planck Institute of Mathematics for its hospitality and stimulating atmosphere; part of this paper was prepared during his stay at the Institute. The second author would like to thank Jacques Hurtubise for his generous encouragement and support during the completion of this paper. She would also like to thank Ron Donagi and Tony Pantev for valuable discussions, Andrei Căldăraru for useful suggestions, and the Department of Mathematics at the University of Pennsylvania for their hospitality, during the preparation of part of this article. Finally, both authors thank the referee as well as Matei Toma for pointing out certain inaccuracies in the original manuscript.

\section{References}

[BPV] W. Barth, C. Peters, and A. Van de Ven, Compact complex surfaces, Springer-Verlag, Berlin, Heidelberg, New York, 1984.

[BBRP] C. Bartocci, U. Bruzzo, D. H. Ruipérez, and J. M. Porras, Mirror symmetry on K3 surfaces via Fourier-Mukai transform, Comm. Math. Phys. 195(1) (1998) 79-93.

[BBDG] K. Becker, M. Becker, K. Dasgupta, and P. S. Green, it Compactification of heterotic theory on non-Kähler complex manifolds: I. Preprint arXiv:hep-th/0301161.

[BH] P. J. Braam and J. Hurtubise, Instantons on Hopf surfaces and monopoles on solid tori, J. Reine Angew. Math. 400 (1989) 146-172.

[BM] T. Bridgeland and A. Maciocia, Fourier-Mukai transforms for $K 3$ and elliptic fibrations, J. Algebraic Geom. 11(4) (2002) 629-657.

[B] T. Bridgeland, Fourier-Mukai transforms for elliptic surfaces, J. Reine Angew. Math. 498 (1998) 115-133.

[Br1] V. Brînzănescu, Néron-Severi group for non-algebraic elliptic surfaces I, elliptic bundle case, Manuscripta Math. 79 (1993) 187-195; II, non-Kählerian case, Manuscripta Math. 84 (1994) 415-420; III, Rev. Roumaine Math. Pures Appl. 43(1-2) (1998) 89-95.

[Br2] Holomorphic vector bundles over compact complex surfaces, Lect. Notes in Math. 1624, Springer, 1996.

[BrMo1] V. Brînzănescu and R. Moraru, Holomorphic rank-2 vector bundles on non-Kähler elliptic surfaces, Ann. Inst. Fourier (Grenoble) 55 (2005) 1659-1683.

[BrMo2] Stable bundles on non-Kähler elliptic surfaces, Comm. Math. Phys. 254 (2005) $565-580$.

[BrU] V. Brînzănescu and K. Ueno, Néron-Severi group for torus quasi bundles over curves. Moduli of vector bundles (Sanda, 1994; Kyoto, 1994), Lecture Notes in Pure and Appl. Math. 179, Dekker, New York, 1996, 11-32.

[C1] A. Căldăraru, Derived categories of twisted sheaves on Calabi-Yau manifolds, PhD thesis, Cornell University, 2000.

[C2] _ Derived categories of twisted sheaves on elliptic threefolds, J. Reine Angew. Math. 544 (2002) 161-179.

[CCFLMZ] G. L. Cardoso, G. Curio, G. Dall'Agata, D. Lüst, P. Manousselis and G. Zoupanos, NonKähler string backgrounds and their five torsion classes. Preprint arXiv:hep-th/0211118.

[DP] R. Donagi and T. Pantev, Torus fibrations, gerbes, and duality, math.AG/0306213.

[F] R. Friedman, Algebraic Surfaces and Holomorphic Vector Bundles, UTX, Springer, New York Berlin Heidelberg, 1998.

[FMW] R. Friedman, J. Morgan, and E. Witten, Vector bundles over elliptic fibrations, J. Algebraic Geom. 2 (1999) 279-401.

[GP] E. Goldstein anf S. Prokushkin, Geometric model for complex non-Kähler manifolds with SU(3) structure. Preprint arXiv:hep-th/0212307. 
[K] K. Kodaira, On the structure of compact complex analytic surfaces I, Amer. J. Math. 86 (1964) 751-798.

[Mo] R. Moraru, Integrable systems associated to a Hopf surface, Canad. J. Math. 55 (3) (2003) 609-635.

[Mu] S. Mukai, Duality between $D(X)$ and $D(\hat{X})$ with its application to Picard sheaves, Nagoya Math. J. 81 (1981) 153-175.

[Mum] D. Mumford, Prym varieties I. Contributions to analysis, Acad. Press, 1974, 325-350.

Institute of Mathematics "Simion Stollow", Romanian Academy, P.O.Box 1-764, RO70700, Bucharest, Romania

E-mail address: Vasile.Brinzanescu@imar.ro

Department of Pure Mathematics, University of Waterloo, 200 University Avenue West, Waterloo, Ontario, Canada N2L 3G1

E-mail address: moraru@math.uwaterloo.ca 